\title{
Value and Risks of the New Quinolones
}

The fluorinated quinolones were newly developed and introduced into therapy a few years ago, and their therapeutic properties are undisputed. However, the sideeffects reported to date in the private medical information service, their significance and causal connection with therapy, have become the source of a controversial discussion in the popular press and on television.

The starting point of the discussion was the profile of sideeffects displayed by the first representative of the quinolone class, nalidixic acid, which is described in Goodman and Gilman's well known text book "Pharmacological Basis of Therapeutics". The range of action and the type and frequency of side-effects of the new fluorinated quinolones, however, differ in many respects. In spite of this, reports on side-effects affecting the central nervous system have recently moved into the foreground of the public discussion, and a causal connection between the event observed and the medication administered is assumed in all cases. Seizures, hallucinations and psychotic reactions are, by their very nature, disturbing for patient and doctor alike. The incidence of seizures, hallucinations and psychotic reactions together following treatment with fluorinated quinolones is, according to present knowledge, 3 in 200,000 cases treated. As a precautionary measure, therefore, patients with a history of convulsions or psychotic symptoms should not be treated with quinolones. The package leaflet of ofloxacin states this with regard to epilepsy. However, a clear connection to any of the CNS disturbances mentioned has yet to be proven.
It is important to mention at this point that the "Medical Letter" of 29th August 1986 contained a list of numerous, chemically unrelated substances for which psychotic reactions had been reported. The list includes some frequently used antimicrobial agents. Ofloxacin and norfloxacin are the new quinolones whose side-effects are best known worldwide at the moment; the other derivatives have so far only been used in a few countries. The comparatively low incidence of allergy to the new quinolones, in addition to the good gastrointestinal tolerance were particularly noteworthy.

Whilst there is usually an obvious relationship between a drug and its desired effects, considerable difficulties arise when assessing the causality of side-effects - particularly since methods of documenting adverse reactions vary so widely. In addition to frequency, it is the type or severity of the side-effect that needs particular consideration.

All points taken into account, i.e. the spectrum of activity, the curative effect and the overall low incidence of side-effects, the value of certain preparations of the new fluorinated quinolones which are considered the first choice drug in some infections clearly outweighs the risks on the basis of present knowledge. For decades now, the aim when developing new preparations has been to achieve maximum efficacy with a minimum of side-effects. As yet there has been no reason to doubt that this has indeed been achieved with most of these new derivatives.

H. Knothe 